\title{
Isolation and identification of Vibrio sp. in the Hepatopancreas of cultured white pacific shrimp (Litopenaeus vannamei)
}

\author{
S. Shanmugasundaram*, P. Mayavu, T. Manikandarajan, M. Suriya, \\ A. Eswar, R. Anbarasu
}

\begin{abstract}
Centre of Advanced Study in Marine Biology, Faculty of Marine Sciences, Annamalai University, Parangipettai - 608 502, Tamil Nadu, India.
\end{abstract}

E-mail address: shanmugasundara89@gmail.com

Keywords: Identification, Pathogens, Penaeus vannamei, Vibrio spp.

\begin{abstract}
The Vibrio is the most common genera with crustaceans often causing various diseases in Aquaculture and significant economic losses. Many Vibrio species are pathogenic to human and have been implicated in food borne diseases. The present study was carried out, the isolation and identification of pathogenic bacterial flora were isolated from infected in hepatopancreas of vannamei. The SPDS Oceanic farm, RS Aqua farm of and Valli vilas Aqua farm Vellar estuary, Cuddalore District, Tamil Nadu, during the period of (September 2013 to November 2013). The collected samples were plated on TCBS- (Thiosulfate-Citrate-Bile salt-Sucrose) agar medium. The present study, totally 253 green colonies were isolated from TCBS agar plates and among these, 175 colonies were identified by using the biochemical tests showed the $V$. parahaemolyticus, $V$. mimicus, $V$. vulnificus, $V$. damsela and $P$. shigelloides. The maximum species was recorded in $V$. parahaemolyticus $(83.4 \%)$ and minimum was observed in $V$. mimicus $(1.7 \%)$.
\end{abstract}

\section{INTRODUCTION}

Aquaculture is the world's fastest growing food production sector, regarding cultured shrimp and prawn the annual rate reach up to $16.8 \%$ between 1984 and 1995 [1]. Shrimp cultivation is one of these most economically important agricultural activities in Asia and South America, and also practiced on worldwide [2]. In international trade, the most prominent product from aquaculture is marine shrimp. Which approximately $26 \%$ of the total product comes from pond-reared Penaeid species [3]. P. vannamei and P. monodon (taxonomy according to [4] are the predominant species of farm-raised shrimp cultivated in both the Eastern and the Western hemispheres. As with any monoculture, raising shrimp in ponds in close proximity to each other increases the spread of diseases [5]. However, shrimp cultivation have been faced many serious problems such as shrimp diseases, unsatisfactory practices, i.e. inadequate control of water quality, etc. The most severe diseases of shrimp, causing the greatest economic losses to growers, are caused by Viruses and Bacteria [6].This kind of diseases attacked due to bacterial infections, particularly caused by luminous Vibrio. [7].Vibrio is well recognized as significance of disease and mortality. Two species, $V$. cholerae and $V$. parahaemolyticus are well documented as human pathogens. It also causes several fish diseases with serious problems for a wide range of wild and farmed species [8].

Pathogenic bacteria have also been involved in this crisis vibrio species are among the most important bacterial pathogens of cultured shrimp, responsible for up to $100 \%$ stricken, species such as $V$. harveyi, $V$. anguillarum, V. parahaemolyticus and $V$. vulnificus have been frequently associated with mortalities both in hatcheries and grow out ponds [9, 10]. The trend of above, the present investigation has been made on isolation and identification of Vibrio species from infected hepatopancreas of P. vannamei. 


\section{MATERIALS AND METHODS}

\section{Collection of samples}

In the present study the infected shrimp samples were collected at three shrimp aquaculture farms (SPDS, RS and Valli vilas) which are located at southern and northern banks of Vellar estuary, Parangipettai, India. During the period of March to May 2014. The collected samples were transferred to laboratory and stored at refrigerator for further analysis.

\section{Isolation of shrimp pathogenic Bacterial colonies:}

The hepatopancreas of infected shrimp was dissected and removed for pathogenic bacteria isolation. The samples are serially diluted $10^{-1}$ to $10^{-9}$ factor. $0.1 \mathrm{ml}$ of samples from $10^{-5}, 10^{-6}$ and $10^{-7}$ were sucked and spread over the sterilized TCBS- (Thiosulfate -Citrate- Bile salt-Sucrose) medium of Peptone-10.00g, Yeast extract- 5.00g, Sodium citrate- 10.0g, Sodium thiasulphate$10.0 \mathrm{~g}$, Sodium cholate- $3.0 \mathrm{~g}$, Oxgall- $5.00 \mathrm{~g}$, Sucrose- $20.0 \mathrm{~g}$, Sodium chloride- $10.0 \mathrm{~g}$, Ferric citrate$1.00 \mathrm{~g}$, Bromo thymolblue-0.04g, Thymol blue- $0.04 \mathrm{~g}$, Agar- $14.00 \mathrm{~g}$, Final $\mathrm{pH}\left(\right.$ at $\left.20^{\circ} \mathrm{C}\right) 8.6 \pm 0.1$ and $1000 \mathrm{ml}$ distilled water and the petri plated are placed overnight at $37-45^{\circ} \mathrm{C}$ at incubator. The incubation period was maintained for 24 to 48 hours at $37^{\circ} \mathrm{C}$ for the pathogenic culture.

\section{Purification and conservation of isolates}

For the Vibrio species identification of each sample, 115 yellow colonies and 253 green colonies were selected form TCBS plates. A total of 175 green colonies were isolated then purified and stored in TCBS agar slant for further studies.

\section{Biochemical identification of shrimp bacteria}

The isolated bacterial species were identified by the following the morphological and biochemical characteristics of the individual colony was recorded. The individual colony was transferred to nutrient agar. The isolates were subjected to following different biochemical test such as Gram staining, Motility, Morphology, Indole test, Methyl red test, Pigmentation test, Voges proskauer test, Citrate test, Urease test, Lactose test, $\mathrm{H}_{2} \mathrm{~S}$ production and starch hydrolysis as described by [11].

\section{RESULTS AND DISCUSSION}

In the present investigation, totally 368 pathogenic bacterial colonies were isolated from infected hepatopancreas of P. vannamei,of which 253 green colonies and 175 yellow colonies (Table 1). In this study was showed $V$. parahaemolyticus, $P$. shigelloides, $V$. damsela, $V$. mimucus and $V$. vulnificus were record. The maximum was recorded in V. parahaemolyticus $(83.4 \%)$ and minimum was recorded of $V$. mimicus $(1.7 \%$ ) (Table 2$)$.

Similar works were done by many researchers on other aquaculture farms [12].Have surveyed $P$. monodon culture ponds of coastal Andhra Pradesh and isolated six species of Vibrios viz., $V$. harveyi, $V$. parahaemolyticus, $V$. alginolyticus, $V$. anguillarum, $V$. vulnificus and $V$. splendidus. Farm-made feeds showed a high incidence of $V$. parahaemolyticus, $V$. cholerae, $E$. coli and Staphylococcus aureus [13].Five species of Vibrio viz., V. alginolyticus, V. parahaemolyticus, $V$. vulnificus, $V$. fluvialis and $V$. mimicus were detected in the pond water and the prawn body with $V$. alginolyticus and $V$. parahaemolyticus as the dominant species for all ponds [14]. [15] Isolated 143 $V$. cholerae non $\mathrm{O} 1$ strains from shrimp farms in Thailand. Mass mortalities due to red disease from $V$. parahaemolyticus along with other Vibrio spp. were isolated by from ponds in the Philippines [16].The number of Vibrio in farm sediment was reported to be 10 to 20 times higher than those in water column [17]. The dominant species $(47.5 \%)$ belonged to the genus Vibrio in water samples from P. monodon pond in Taiwan [18]. Severe stress and injury to shrimp under poor environmental conditions lower their resistance, rendering them susceptible to viral as well as bacterial infection [19]. Vibriosis is known to affect a wide range of fish and shellfish organisms $[20,21]$.In the present study bacterial species were isolated from infected shrimp muscle tissues. 
Epizoobiology and pathogenicity of bacterial infections caused by many problems in cultured giant tiger prawn Penaeus monodon [22]. Vibrio species are considered as part of the normal flora of seawater and can invade marine animals [23]. [24] Has found total Vibrio loads in healthy and infected shrimp during a complete 60-day culture cycle. However, sick shrimp presented an increase in luminescent vibrios suggesting that infection involves the multiplication of a specific population of pathogens

\section{CONCLUSSION:}

The present study revealed that the pathogenic bacteria are perhaps those most important pathogens in shrimp culture ponds causing several mortalities and financial losses. There is also a need to develop the shrimp culture practice and control the pathogenic microbes is very essential.

Table: 1 SPDS, Oceana farms from yellow and green colonies were isolated.

\begin{tabular}{ccccccc}
\hline $\begin{array}{c}\text { SPDS, } \\
\text { Oceana } \\
\text { farms }\end{array}$ & $\begin{array}{c}\text { Serial } \\
\text { dilution } \\
\mathbf{1 0}^{-5}\end{array}$ & $\begin{array}{c}\text { Serial } \\
\text { dilution } \mathbf{1 0}^{-\mathbf{6}}\end{array}$ & $\begin{array}{c}\text { Serial } \\
\text { dilution } \mathbf{1 0}^{-\mathbf{7}}\end{array}$ & $\begin{array}{c}\text { Total } \\
\text { vibrio } \\
\text { colonies }\end{array}$ & $\begin{array}{c}\text { Yellow } \\
\text { Colonies }\end{array}$ & $\begin{array}{c}\text { Green } \\
\text { Colonies }\end{array}$ \\
\hline Pond 1 & 30 & 12 & 3 & 35 & 14 & 21 \\
Pond 2 & 16 & 7 & 2 & 25 & 11 & 14 \\
Pond 3 & 32 & 8 & 1 & 41 & 18 & 23 \\
Pond 4 & 10 & 4 & 0 & 14 & 5 & 9 \\
Pond 5 & 21 & 13 & 4 & 38 & 7 & 31 \\
Total & & & & $\mathbf{1 5 3}$ & $\mathbf{5 5}$ & $\mathbf{9 8}$ \\
\hline
\end{tabular}

Table 2: RS Aqua farms from yellow and green colonies were isolated.

\begin{tabular}{|c|c|c|c|c|c|c|}
\hline $\begin{array}{l}\text { RS Aqua } \\
\text { farms }\end{array}$ & $\begin{array}{c}\text { Serial dilution } \\
10^{-5} \\
\end{array}$ & $\begin{array}{c}\text { Serial dilution } \\
10^{-6} \\
\end{array}$ & $\begin{array}{c}\text { Serial } \\
\text { dilution } 10^{-7} \\
\end{array}$ & $\begin{array}{c}\text { Total vibrio } \\
\text { colonies }\end{array}$ & $\begin{array}{c}\text { Yellow } \\
\text { colonies }\end{array}$ & $\begin{array}{c}\text { Green } \\
\text { colonies }\end{array}$ \\
\hline Pond 1 & 31 & 10 & 4 & 45 & 10 & 35 \\
\hline Pond 2 & 28 & 16 & 2 & 46 & 17 & 29 \\
\hline Pond 3 & 16 & 8 & 1 & 25 & 12 & 13 \\
\hline \multicolumn{4}{|c|}{ Total } & 116 & 39 & 77 \\
\hline
\end{tabular}

Table 3: Valli vilas farms from yellow and green colonies were isolated.

\begin{tabular}{|c|c|c|c|c|c|c|}
\hline $\begin{array}{l}\text { SPDS, Oceana } \\
\text { farms }\end{array}$ & $\begin{array}{c}\text { Serial dilution } \\
10^{-5}\end{array}$ & $\begin{array}{l}\text { Serial dilution } \\
10^{-6}\end{array}$ & $\begin{array}{c}\text { Serial dilution } \\
10^{-7}\end{array}$ & $\begin{array}{c}\text { Total vibrio } \\
\text { colonies }\end{array}$ & $\begin{array}{l}\text { Yellow } \\
\text { Colonies }\end{array}$ & $\begin{array}{c}\text { Green } \\
\text { Colonies }\end{array}$ \\
\hline Pond 1 & 32 & 15 & 1 & 48 & 8 & 40 \\
\hline Pond 2 & 33 & 17 & 0 & 51 & 13 & 38 \\
\hline \multicolumn{4}{|c|}{ Total } & 99 & 21 & 78 \\
\hline
\end{tabular}




\begin{tabular}{|c|c|c|c|c|c|c|c|c|c|c|c|c|c|}
\hline 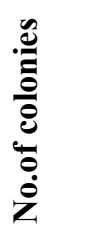 & 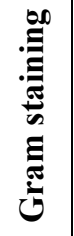 & & 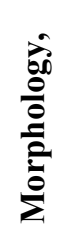 & $\begin{array}{l}\frac{\varrho}{0} \\
\stackrel{\Xi}{\Xi}\end{array}$ & 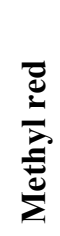 & 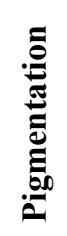 & 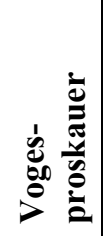 & : & 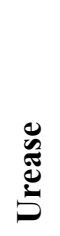 & 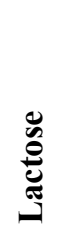 & 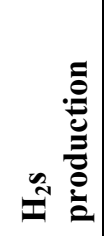 & 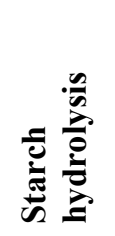 & 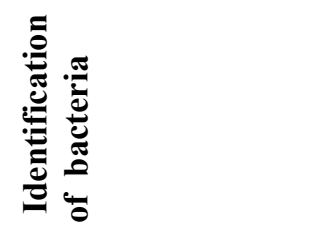 \\
\hline Ss1 & - & + & rod & + & + & $\mathrm{G}$ & - & + & + & - & - & - & V.vulnificus \\
\hline Ss2 & - & + & rod & + & + & $\mathrm{G}$ & - & - & - & - & - & + & V.parahaemolyticus \\
\hline Ss3 & - & + & rod & + & + & $\mathrm{G}$ & - & - & - & - & - & + & V. parahaemolyticus \\
\hline Ss4 & - & + & rod & + & + & $\mathrm{G}$ & - & - & - & - & - & + & V. parahaemolyticus \\
\hline Ss5 & - & + & rod & + & + & $\mathrm{G}$ & - & - & - & - & - & + & V. parahaemolyticus \\
\hline Ss6 & - & + & rod & + & + & $\mathrm{G}$ & - & - & - & - & - & + & V. parahaemolyticus \\
\hline Ss7 & - & + & rod & + & + & $\mathrm{G}$ & - & - & - & - & - & + & V. parahaemolyticus \\
\hline Ss8 & - & + & rod & + & + & $\mathrm{G}$ & - & - & - & - & - & + & V. parahaemolyticus \\
\hline Ss9 & - & + & rod & + & + & $\mathrm{G}$ & - & - & - & - & - & + & V. parahaemolyticus \\
\hline Ss10 & - & + & rod & + & + & $\mathrm{G}$ & - & - & - & - & - & + & V. parahaemolyticus \\
\hline Ss11 & - & + & rod & + & + & $\mathrm{G}$ & - & - & + & - & - & - & P.shigelloides \\
\hline Ss12 & - & + & rod & + & + & $\mathrm{G}$ & - & - & - & - & - & + & V. parahaemolyticus \\
\hline Ss13 & - & + & rod & + & + & $\mathrm{G}$ & - & - & - & - & - & + & V. parahaemolyticus \\
\hline Ss14 & - & + & rod & + & + & $\mathrm{G}$ & - & - & - & - & - & + & V.parahaemolyticus \\
\hline Ss 15 & - & + & rod & + & + & $\mathrm{G}$ & - & + & + & - & - & - & V.vulnificus \\
\hline Ss16 & - & + & rod & + & + & $\mathrm{G}$ & - & + & + & - & - & - & V.vulnificus \\
\hline Ss17 & - & + & rod & - & + & $\mathrm{G}$ & + & - & + & - & - & + & V.damsela \\
\hline Ss18 & - & + & rod & + & + & $\mathrm{G}$ & - & - & - & - & - & + & V. parahaemolyticus \\
\hline Ss19 & - & + & rod & + & + & G & - & - & - & - & - & + & V. parahaemolyticus \\
\hline Ss20 & - & + & rod & + & + & $\mathrm{G}$ & - & - & - & - & - & + & V.parahaemolyticus \\
\hline Ss21 & - & + & rod & + & + & $\mathrm{G}$ & - & - & - & - & - & + & V.parahaemolyticus \\
\hline Ss22 & - & + & rod & + & + & G & - & - & - & - & - & + & V. parahaemolyticus \\
\hline Ss23 & - & + & rod & + & + & $\mathrm{G}$ & - & - & - & - & - & + & V. parahaemolyticus \\
\hline Ss24 & - & + & rod & + & + & $\mathrm{G}$ & - & - & - & - & - & + & V. parahaemolyticus \\
\hline Ss25 & - & + & rod & + & + & $\mathrm{G}$ & - & - & - & - & - & + & V.parahaemolyticus \\
\hline Ss26 & - & + & rod & + & + & $\mathrm{G}$ & - & - & - & - & - & + & V. parahaemolyticus \\
\hline Ss27 & - & + & rod & + & + & $\mathrm{G}$ & - & - & - & - & - & + & V.parahaemolyticus \\
\hline Ss28 & - & + & rod & + & + & $\mathrm{G}$ & - & - & - & - & - & + & V.parahaemolyticus \\
\hline Ss29 & - & + & rod & + & + & $\mathrm{G}$ & - & - & - & - & - & + & V. parahaemolyticus \\
\hline Ss30 & - & + & rod & + & + & $\mathrm{G}$ & - & - & - & - & - & + & V.parahaemolyticus \\
\hline Ss31 & - & + & rod & + & + & $\mathrm{G}$ & - & - & - & - & - & + & V.parahaemolyticus \\
\hline Ss32 & - & + & rod & + & + & $\mathrm{G}$ & - & - & - & - & - & + & V. parahaemolyticus \\
\hline Ss33 & - & + & rod & + & + & $\mathrm{G}$ & - & - & - & - & - & + & V.parahaemolyticus \\
\hline Ss34 & - & + & rod & + & + & $\mathrm{G}$ & - & - & - & - & - & + & V.parahaemolyticus \\
\hline Ss35 & - & + & $\operatorname{rod}$ & + & + & $\mathrm{G}$ & - & - & - & - & - & + & V. parahaemolyticus \\
\hline Ss36 & - & + & rod & + & + & $\mathrm{G}$ & - & - & - & - & - & + & V.parahaemolyticus \\
\hline Ss37 & - & + & rod & + & + & $\mathrm{G}$ & - & - & - & - & - & + & V.parahaemolyticus \\
\hline Ss38 & - & + & rod & + & + & $\mathrm{G}$ & - & - & - & - & - & + & V. parahaemolyticus \\
\hline Ss39 & - & + & rod & + & + & $\mathrm{G}$ & - & - & - & - & - & + & V.parahaemolyticus \\
\hline Ss40 & - & + & rod & + & + & $\mathrm{G}$ & - & - & - & - & - & + & V. parahaemolyticus \\
\hline Ss41 & - & + & rod & & & $\mathrm{G}$ & - & - & - & - & - & + & V.mimicus \\
\hline Ss42 & - & + & rod & + & + & $\mathrm{G}$ & - & - & - & - & - & + & V. parahaemolyticus \\
\hline Ss43 & - & + & rod & + & + & $\mathrm{G}$ & - & - & - & - & - & + & V. parahaemolyticus \\
\hline Ss44 & - & + & rod & + & + & $\mathrm{G}$ & - & - & - & - & - & + & V.parahaemolyticus \\
\hline Ss45 & - & + & rod & + & + & $\mathrm{G}$ & - & - & - & - & - & + & V. parahaemolyticus \\
\hline Ss46 & - & + & rod & + & + & $\mathrm{G}$ & - & - & - & - & - & + & V. parahaemolyticus \\
\hline Ss47 & - & + & rod & + & + & $\mathrm{G}$ & - & - & - & - & - & + & V.parahaemolyticus \\
\hline Ss48 & - & + & rod & + & + & $\mathrm{G}$ & - & - & - & - & - & + & V. parahaemolyticus \\
\hline Ss49 & - & + & rod & + & + & $\mathrm{G}$ & - & - & - & - & - & + & V.parahaemolyticus \\
\hline Ss50 & - & + & rod & + & + & $\mathrm{G}$ & - & - & - & - & - & + & V.parahaemolyticus \\
\hline Ss51 & - & + & rod & + & + & $\mathrm{G}$ & - & - & - & - & - & + & V. parahaemolyticus \\
\hline Ss52 & - & + & rod & + & + & $\mathrm{G}$ & - & - & - & - & - & + & V.parahaemolyticus \\
\hline Ss53 & - & + & rod & + & + & $\mathrm{G}$ & - & - & - & - & - & + & V. parahaemolyticus \\
\hline Ss54 & - & + & rod & + & + & $\mathrm{G}$ & - & - & - & - & - & + & V. parahaemolyticus \\
\hline
\end{tabular}




\begin{tabular}{|c|c|c|c|c|c|c|c|c|c|c|c|c|c|}
\hline Ss55 & - & + & rod & + & + & $\mathrm{G}$ & - & - & - & - & - & + & V. parahaemolyticus \\
\hline Ss56 & - & + & rod & + & + & $\mathrm{G}$ & - & - & - & - & - & + & V. parahaemolyticus \\
\hline Ss57 & - & + & rod & + & + & $\mathrm{G}$ & - & - & - & - & - & + & V.parahaemolyticus \\
\hline Ss58 & - & + & rod & + & + & $\mathrm{G}$ & - & - & - & - & - & + & V.parahaemolyticus \\
\hline Ss59 & - & + & rod & + & + & $\mathrm{G}$ & - & - & - & - & - & + & V. parahaemolyticus \\
\hline Ss60 & - & + & rod & + & + & $\mathrm{G}$ & - & - & - & - & - & + & V. parahaemolyticus \\
\hline Ss61 & - & + & rod & + & + & $\mathrm{G}$ & - & - & - & - & - & + & V.parahaemolyticus \\
\hline Ss62 & - & + & rod & + & + & $\mathrm{G}$ & - & - & - & - & - & + & V.parahaemolyticus \\
\hline Ss63 & - & + & rod & + & + & $\mathrm{G}$ & - & - & - & - & - & + & V. parahaemolyticus \\
\hline Ss64 & - & + & rod & + & + & $\mathrm{G}$ & - & - & - & - & - & + & V. parahaemolyticus \\
\hline Ss65 & - & + & rod & + & + & $\mathrm{G}$ & - & - & - & - & - & + & V.parahaemolyticus \\
\hline Ss66 & - & + & rod & + & + & $\mathrm{G}$ & - & - & - & - & - & + & V.parahaemolyticus \\
\hline Ss67 & - & + & rod & + & + & $\mathrm{G}$ & - & - & - & - & - & + & V.parahaemolyticus \\
\hline Ss68 & - & + & rod & + & + & $\mathrm{G}$ & - & - & - & - & - & + & V. parahaemolyticus \\
\hline Ss69 & - & + & rod & + & + & $\mathrm{G}$ & - & - & - & - & - & + & V. parahaemolyticus \\
\hline Ss 80 & - & + & rod & + & + & $\mathrm{G}$ & - & - & - & - & - & + & V. parahaemolyticus \\
\hline Ss81 & - & + & rod & + & + & $\mathrm{G}$ & - & - & - & - & - & + & V.parahaemolyticus \\
\hline Ss82 & - & + & rod & + & + & $\mathrm{G}$ & - & - & - & - & - & + & V.parahaemolyticus \\
\hline Ss83 & - & + & rod & + & + & $\mathrm{G}$ & - & - & - & - & - & + & V. parahaemolyticus \\
\hline Ss84 & - & + & rod & + & + & $\mathrm{G}$ & - & - & - & - & - & + & V. parahaemolyticus \\
\hline Ss85 & - & + & rod & + & + & $\mathrm{G}$ & - & - & - & - & - & + & V. parahaemolyticus \\
\hline Ss86 & - & + & rod & + & + & $\mathrm{G}$ & - & - & - & - & - & + & V. parahaemolyticus \\
\hline Ss87 & - & + & rod & + & + & $\mathrm{G}$ & - & - & - & - & - & + & V.parahaemolyticus \\
\hline Ss88 & - & + & rod & + & + & $\mathrm{G}$ & - & - & - & - & - & + & V. parahaemolyticus \\
\hline Ss89 & - & + & rod & + & + & $\mathrm{G}$ & - & - & - & - & - & + & V. parahaemolyticus \\
\hline Ss90 & - & + & rod & + & + & $\mathrm{G}$ & - & - & - & - & - & + & V.parahaemolyticus \\
\hline Ss91 & - & + & rod & + & + & $\mathrm{G}$ & - & - & - & - & - & + & V. parahaemolyticus \\
\hline Ss92 & - & + & rod & + & + & $\mathrm{G}$ & - & - & - & - & - & + & V. parahaemolyticus \\
\hline Ss93 & - & + & rod & + & + & $\mathrm{G}$ & - & - & - & - & - & + & V. parahaemolyticus \\
\hline Ss94 & - & + & rod & + & + & $\mathrm{G}$ & - & - & - & - & - & + & V. parahaemolyticus \\
\hline Ss95 & - & + & rod & + & + & $\mathrm{G}$ & - & - & - & - & - & + & V. parahaemolyticus \\
\hline Ss96 & - & + & rod & + & + & $\mathrm{G}$ & - & - & - & - & - & + & V. parahaemolyticus \\
\hline Ss97 & - & + & rod & + & + & $\mathrm{G}$ & - & + & + & - & - & - & V.vulnificus \\
\hline Ss98 & - & + & rod & + & + & $\mathrm{G}$ & - & + & + & - & - & - & V.vulnificus \\
\hline Ss99 & - & + & rod & & & $\mathrm{G}$ & + & - & + & - & - & + & V.damsela \\
\hline Ss100 & - & + & rod & + & + & $\mathrm{G}$ & - & - & - & - & - & + & V. parahaemolyticus \\
\hline Ss101 & - & + & rod & + & + & $\mathrm{G}$ & - & - & - & - & - & + & V.parahaemolyticus \\
\hline Ss102 & - & + & rod & & & $\mathrm{G}$ & - & - & - & - & - & - & V.mimicus \\
\hline Ss103 & - & + & rod & + & + & $\mathrm{G}$ & - & - & - & - & - & + & V. parahaemolyticus \\
\hline Ss104 & - & + & rod & + & + & $\mathrm{G}$ & - & - & - & - & - & + & V. parahaemolyticus \\
\hline Ss105 & - & + & rod & & & $\mathrm{G}$ & - & & & - & & & V.mimicus \\
\hline Ss106 & - & + & rod & + & + & $\mathrm{G}$ & - & - & - & - & - & + & V.parahaemolyticus \\
\hline Ss107 & - & + & rod & + & + & $\mathrm{G}$ & - & - & - & - & - & + & V. parahaemolyticus \\
\hline Ss108 & - & + & rod & + & + & $\mathrm{G}$ & - & + & + & - & - & - & V.vulnificus \\
\hline Ss109 & - & + & rod & + & + & $\mathrm{G}$ & - & + & + & - & - & - & V.vulnificus \\
\hline Ss110 & - & + & rod & + & + & $\mathrm{G}$ & - & + & + & - & - & - & V.vulnificus \\
\hline Ss111 & - & + & rod & + & + & $\mathrm{G}$ & - & + & + & - & - & - & V.vulnificus \\
\hline Ss112 & - & + & rod & + & + & $\mathrm{G}$ & - & - & + & - & - & - & P.shigelloides \\
\hline Ss113 & - & + & rod & + & + & $\mathrm{G}$ & - & - & + & - & - & - & P.shigelloides \\
\hline Ss114 & - & + & rod & + & + & $\mathrm{G}$ & - & - & + & - & - & - & P.shigelloides \\
\hline Ss115 & - & + & rod & + & + & $\mathrm{G}$ & - & - & - & - & - & + & V.parahaemolyticus \\
\hline Ss116 & - & + & rod & + & + & $\mathrm{G}$ & - & - & - & - & - & + & V. parahaemolyticus \\
\hline Ss117 & - & + & rod & + & + & $\mathrm{G}$ & - & - & - & - & - & + & V. parahaemolyticus \\
\hline Ss118 & - & + & rod & + & + & $\mathrm{G}$ & - & - & - & - & - & + & V.parahaemolyticus \\
\hline Ss119 & - & + & rod & + & + & $\mathrm{G}$ & - & - & - & - & - & + & V. parahaemolyticus \\
\hline Ss120 & - & + & rod & + & + & $\mathrm{G}$ & - & - & - & - & - & + & V. parahaemolyticus \\
\hline Ss121 & - & + & rod & + & + & $\mathrm{G}$ & - & - & - & - & - & + & V. parahaemolyticus \\
\hline Ss122 & - & + & rod & + & + & $\mathrm{G}$ & - & - & - & - & - & + & V.parahaemolyticus \\
\hline Ss123 & - & + & rod & + & + & $\mathrm{G}$ & - & - & - & - & - & + & V. parahaemolyticus \\
\hline Ss124 & - & + & rod & + & + & $\mathrm{G}$ & - & - & - & - & - & + & V. parahaemolyticus \\
\hline
\end{tabular}




\begin{tabular}{|c|c|c|c|c|c|c|c|c|c|c|c|c|c|}
\hline Ss125 & - & + & rod & + & + & $\mathrm{G}$ & - & - & - & - & - & + & V. parahaemolyticus \\
\hline Ss126 & - & + & rod & + & + & $\mathrm{G}$ & - & - & - & - & - & + & V.parahaemolyticus \\
\hline Ss127 & - & + & rod & + & + & $\mathrm{G}$ & - & - & - & - & - & + & V.parahaemolyticus \\
\hline Ss128 & - & + & rod & + & + & $\mathrm{G}$ & - & - & - & - & - & + & V. parahaemolyticus \\
\hline Ss129 & - & + & rod & + & + & $\mathrm{G}$ & - & - & - & - & - & + & V.parahaemolyticus \\
\hline Ss130 & - & + & rod & + & + & $\mathrm{G}$ & - & - & - & - & - & + & V.parahaemolyticus \\
\hline Ss131 & - & + & rod & + & + & $\mathrm{G}$ & - & - & - & - & - & + & V.parahaemolyticus \\
\hline Ss132 & - & + & rod & + & + & $\mathrm{G}$ & - & - & - & - & - & + & V.parahaemolyticus \\
\hline $\begin{array}{l}\text { Ss133 } \\
\end{array}$ & - & + & rod & + & + & $\mathrm{G}$ & - & - & - & - & - & + & V. parahaemolyticus \\
\hline Ss134 & - & + & rod & + & + & $\mathrm{G}$ & - & - & - & - & - & + & V.parahaemolyticus \\
\hline Ss135 & - & + & rod & + & + & $\mathrm{G}$ & - & - & + & - & - & - & P.shigelloides \\
\hline Ss136 & - & + & rod & + & + & $\mathrm{G}$ & - & - & + & - & - & - & P.shigelloides \\
\hline Ss137 & - & + & rod & + & + & $\mathrm{G}$ & - & - & + & - & - & - & P.shigelloides \\
\hline Ss138 & - & + & rod & + & + & $\mathrm{G}$ & - & - & + & - & - & - & P.shigelloides \\
\hline Ss139 & - & + & rod & + & + & $\mathrm{G}$ & - & - & + & - & - & - & P.shigelloides \\
\hline Ss140 & - & + & rod & + & + & $\mathrm{G}$ & - & - & + & - & - & - & P.shigelloides \\
\hline Ss141 & - & + & rod & & & $\mathrm{G}$ & + & - & + & - & - & + & V.damsela \\
\hline Ss142 & - & + & rod & & & $\mathrm{G}$ & + & - & + & - & - & + & V.damsela \\
\hline Ss143 & - & + & rod & + & + & $\mathrm{G}$ & - & - & - & - & - & + & V. parahaemolyticus \\
\hline Ss144 & - & + & rod & + & + & $\mathrm{G}$ & - & - & - & - & - & + & V. parahaemolyticus \\
\hline Ss145 & - & + & rod & + & + & $\mathrm{G}$ & - & - & - & - & - & + & V. parahaemolyticus \\
\hline Ss146 & - & + & rod & + & + & $\mathrm{G}$ & - & - & - & - & - & + & V.parahaemolyticus \\
\hline Ss147 & - & + & rod & + & + & $\mathrm{G}$ & - & - & - & - & - & + & V. parahaemolyticus \\
\hline Ss148 & - & + & rod & + & + & $\mathrm{G}$ & - & - & - & - & - & + & V. parahaemolyticus \\
\hline Ss149 & - & + & rod & + & + & $\mathrm{G}$ & - & - & - & - & - & + & V. parahaemolyticus \\
\hline Ss150 & - & + & rod & + & + & $\mathrm{G}$ & - & - & - & - & - & + & V. parahaemolyticus \\
\hline Ss151 & - & + & rod & + & + & $\mathrm{G}$ & - & - & - & - & - & + & V.parahaemolyticus \\
\hline Ss152 & - & + & rod & + & + & $\mathrm{G}$ & - & - & - & - & - & + & V.parahaemolyticus \\
\hline Ss153 & - & + & rod & + & + & $\mathrm{G}$ & - & - & + & - & - & - & P.shigelloides \\
\hline Ss154 & - & + & rod & + & + & $\mathrm{G}$ & - & - & + & - & - & - & P.shigelloides \\
\hline Ss155 & - & + & rod & + & + & $\mathrm{G}$ & - & - & + & - & - & - & P.shigelloides \\
\hline Ss156 & - & + & rod & + & + & $\mathrm{G}$ & - & - & + & - & - & - & P.shigelloides \\
\hline Ss157 & - & + & rod & + & + & $\mathrm{G}$ & - & - & - & - & - & + & V.parahaemolyticus \\
\hline Ss158 & - & + & rod & + & + & $\mathrm{G}$ & - & - & - & - & - & + & V. parahaemolyticus \\
\hline Ss159 & - & + & rod & + & + & $\mathrm{G}$ & - & - & - & - & - & + & V. parahaemolyticus \\
\hline Ss160 & - & + & rod & + & + & $\mathrm{G}$ & - & - & - & - & - & + & V.parahaemolyticus \\
\hline Ss161 & - & + & rod & + & + & $\mathrm{G}$ & - & - & - & - & - & + & V. parahaemolyticus \\
\hline Ss162 & - & + & rod & + & + & $\mathrm{G}$ & - & - & - & - & - & + & V. parahaemolyticus \\
\hline Ss163 & - & + & rod & + & + & $\mathrm{G}$ & - & - & - & - & - & + & V. parahaemolyticus \\
\hline Ss164 & - & + & rod & + & + & $\mathrm{G}$ & - & - & - & - & - & + & V.parahaemolyticus \\
\hline Ss165 & - & + & rod & + & + & $\mathrm{G}$ & - & - & - & - & - & + & V. parahaemolyticus \\
\hline Ss166 & - & + & rod & + & + & G & - & - & - & - & - & + & V. parahaemolyticus \\
\hline Ss167 & - & + & rod & + & + & $\mathrm{G}$ & - & - & - & - & - & + & V.parahaemolyticus \\
\hline Ss168 & - & + & rod & + & + & $\mathrm{G}$ & - & - & - & - & - & + & V. parahaemolyticus \\
\hline Ss169 & - & + & rod & + & + & G & - & - & - & - & - & + & V. parahaemolyticus \\
\hline Ss170 & - & + & rod & + & + & $\mathrm{G}$ & - & - & - & - & - & + & V.parahaemolyticus \\
\hline Ss171 & - & + & rod & + & + & $\mathrm{G}$ & - & - & - & - & - & + & V. parahaemolyticus \\
\hline Ss172 & - & + & rod & + & + & $\mathrm{G}$ & - & - & - & - & - & + & V. parahaemolyticus \\
\hline Ss173 & - & + & rod & + & + & $\mathrm{G}$ & - & - & - & - & - & + & V.parahaemolyticus \\
\hline Ss174 & 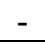 & + & rod & + & + & $\mathrm{G}$ & - & - & - & - & - & + & V. parahaemolyticus \\
\hline Ss175 & - & + & rod & + & + & $\mathrm{G}$ & - & - & - & - & - & + & V.parahaemolyticus \\
\hline
\end{tabular}


Table 4. Biochemical identification of pathogenic bacterial isolate from infected Hepatopancreas vannamei

\begin{tabular}{|l|l|}
\hline Name of the pathogen & \% of vibrio species \\
\hline V.paraheamolyticus & 83.4 \\
\hline V. vulnification & 5.3 \\
\hline V. mimicus & 1.7 \\
\hline V. damsel & 2.2 \\
\hline P.shigelloides & 7.1 \\
\hline
\end{tabular}

\section{ACKNOWLEDGEMENT}

The Authors would like to my Research Supervisor Dr. P.Mayavu, M.Sc., Ph.D., and for help and encouragement, the UGC - New Delhi, India for financial support, internet facility and Laboratory facility provided for in our campus CAS in Marine Biology Annamalai University at Parangipettai, India.

\section{Reference}

[1] Subasinghe R.D, Bartly M, Mcgladdery S, Barg U., Advances in shrimp biotechnology. National Centre for Genetic Engineering and Biotechnology, Bankok, (1998).

[2] Nimrat Subuntith, Bart Amrit N, Keatsaksit Aritsara, Vuthiphandchai Verapong., Aquaculture, (2008) vol. 274, no. 2-4, p. 247-253.

[3] FAO (Food \& Agriculture Organization of the United Nations), the State of World Fisheries and Aquaculture (SOFIA). Rome: FAO, (2002).

[4] Holthuis L.B., FAO Fish Synop. 125. Rome: FAO. (1980).

[5] Kautsky N, Ronnback P, Tedengren M, Troell M., Aquaculture, (2000).191, 145-161.

[6] Lightner D.V, Bell T.A, Redman R.M, Mohney L.L, Natividad J.M, Rukyani A, Poernomo A., In Diseases in Asian Aquaculture, Fish Health Section, Asian Fisheries Society, (1992) pp. 5780 .

[7] Musa N, Wei L.S, Shaharom F, Wee W., World Applied Sciences Journal, (2008) 3(6): 903905.

[8] Damsgard B, sorum U, Ugelstad I, Eliassen R.A, Mortensen A., Aquaculture, (2004) 239, 3746.

[9] Baticados M.C.L, Lavilla-Pitogo C.R, Cruz-Lacierda E.R, de la Pena L.D, Sunaz. N.A., Dis. Aquat. Organ, (1990) 9: 133-139.

[10] Mohney L.L, Lightner D.V, Bell T.A., J world Aquaculr, (1994) 25:116-125.

[11] Jolt J.G, Krieg N.R, Stanley J.T, Williams S.T., Bergey's manual of systematic bacteriology. $9^{\text {th }}$ edn. Maryland: Williams and wilkins Co. Baltimore.(1994) 786.

[12] Jayasree L, Janakiram P, Madhavi R., J. World Aquac.Soc, (2006) 37: 523-532.

[13] Raghavan R.P., Naga World Fish Center Quarterly, (2003) 26: 22-24.

[14] Ni C, Lin Y, Ye D, Zeng H, Yao R, Gu J., J. Oceanogr. Taiwan Straits/Taiwan Haixia, (1995) 14:73-79.

[15] Dalsgaard A, Serichantalergs O, Shimada T, Sethabutr O, Echeverria P., J. Med. Microbiol,(1995) 43: 216-220. 
[16] Tendencia A.E.V, Dureza L.A., Aquaculture, (1997) 154:107-114.

[17] Li Q, Chen B, Qu K, Xin F, Li J, Zhao F, Yan Y., J. Fish.Sci.China, (2002) 9: 367-370.

[18] Wei W.C, Hsu C.H., 6th Asian Fisheries Forum Book of Abstracts, (2001) pp 346, 25-30.

[19] Liu C.I., Fish diseases Proc. R. Jpn. Symp. Taipei, Taiwan, (1990) p. 180-201.

[20] Brock J.A, Lea Master B., World Aquaculture Society, Baton Rouge, Louisiana, USA, (1992) p. 212-226.

[21] Aguirre Guzman G., Programa Nacional de Sanidad Acuicola y la Red de Diagnostico, (2004) 1: $1-9$.

[22] Chen S.N, Huang S.L, Kou G.N., The oceanic institute, (1992) 195-206.

[23] Olsen J.E, Aabo S, Hill W, Notermans S, Wernars K, Granum P.E, Popovic T, Rasmussen H.N, Olsvik O., Inst.J.Food Microbiol, (1995) 28:1-78

[24] Lavilla Pitogo C.R, Leano E.M, Paner M.G., Aquaculture, (1998) 164: 337-349. 